\title{
Treatment of isolated lesions of the posterior cruciate ligament
}

\author{
TRATAMENTO DA LESÃO ISOLADA DO LIGAMENTO CRUZADO POSTERIOR \\ Authorship: Brazilian Society of Orthopedics and Traumatology \\ Participants: Meleiro SAS, Mendes VTA, Kaleka CC, Cury RPL
}

http://dx.doi.org/10.1590/1806-9282.61.02.102

The Guidelines Project, an initiative of the Brazilian Medical Association, aims to combine information from the medical field in order to standardize procedures to assist the reasoning and decision-making of doctors.

The information provided through this project must be assessed and criticized by the physician responsible for the conduct that will be adopted, depending on the conditions and the clinical status of each patient.

\section{Description of the eVIDENCE COLlection METHOD}

To develop this guideline the following primary electronic databases were consulted: Medline (1966 to 2012) via PubMed and LILACS. The search for evidence came from actual clinical scenarios and used keywords (MeSH terms): "Humans", "Adult", "Knee", "Posterior Cruciate Ligament", "Posterior Cruciate Ligament/surgery*" "Posterior Cruciate Ligament/pathology*", "Posterior Cruciate Ligament/injuries*”, "Arthroscopic Surgeries", "Allograft”, "Rehabilitation", "Treatment Outcome”, "Outcome Assessment", "Prospective Studies". The articles were selected after critical evaluation of the strength of scientific evidence by experts in related orthopedic specialties, and publications of greatest strength were used for recommendation. The recommendations were drawn from group discussion. The entire guideline was reviewed by an independent group, specializing in evidence-based clinical guidelines.

\section{Grade OF ReCommendation AND STRENGTH OF EVIDENCE}

A. Experimental or observational studies of higher consistency.

B. Experimental or observational studies of lower consistency.

C. Case reports (non-controlled studies).

D. Opinions without critical evaluation, based on consensus, physiological studies, or animal models.

\section{Objectives}

This guideline aims to guide the treatment of isolated lesions of the posterior cruciate ligament in adult patients.

\section{Conflict of Interest}

No conflict of interest informed.

\section{INTRODUCTION}

The posterior cruciate ligament (PCL) is the main restrictor of posterior tibial translation and acts as a secondary stabilizer for varization, valgization, and lateral and medial rotation of this bone $(\mathbf{D}){ }^{1-4}$ It is functionally divided into two bundles, the anterolateral (AL) band and the posteromedial (PM) band, named according to the direction taken by the bundles in the path between the tibia and the femur and its insertion into the bone..$^{1-5}$ With the knee in flexion, the AL bundle is tight and the PM loose, while with the knee in extension, the tensions are reversed (D). ${ }^{1-5}$

PCL injuries are far less frequent than other knee ligament injuries (D) ${ }^{1-4,6}$ The incidence of these injuries in the general population is around 3\%, rising sharply in the population susceptible to trauma, reaching $37 \%$ (D) ${ }^{1-3,6}$ mainly in car accidents and high-energy incidents, where a high prevalence of associated injuries is observed $(\mathbf{D}) \cdot{ }^{1,6}$ Among the population of athletes, there is also a higher incidence of PCL injuries than in the general population, but this difference varies depending on the type of activity practiced $(\mathbf{D})^{1,6}(\mathbf{B}) .^{7}$ Isolated PCL injuries are less common than associated injuries $(\mathbf{D})^{1,3}(\mathbf{B}) .^{7}$

The main mechanism involved in general PCL injuries derives from direct trauma to the tibia in the anteroposterior direction with the knee in flexion (D). ${ }^{1,3,5}$ In sports, the main mechanism of injury is excessive flexion of the knees, caused by falls with knees flexed, for example $(\mathbf{D}){ }^{5}$

PCL injuries are usually classified into three different levels according to the magnitude of the posterior translation of the tibia relative to the femur: grade I - 1 to 5 $\mathrm{mm}$; grade II - 5 to $10 \mathrm{~mm}$; and grade III - greater than $10 \mathrm{~mm}(\mathbf{D}) \cdot 3,5$

The treatment of isolated PCL injury represents a dilemma for orthopedists. Although there are well-described treatment protocols for injuries of the anterior cruciate 
ligament and collateral ligaments of the knee, there is no consensus regarding the treatment of isolated PCL injury, and little evidence-based information to guide the management of this injury (D). ${ }^{6}$ While some patients remain asymptomatic and return to the sport, others present dysfunction and progression to degeneration of the knee joint.

Due to the lack of randomized studies for comparing the results, much debate is created regarding indications and types of treatment, in addition to follow-up (D) ${ }^{3,6}$ The suspicion, investigation and recognition of this injury are of fundamental importance to guide patients to ideal treatment.

The purpose of this guideline is to review the latest treatments for isolated PCL injury, disclosing the surgical indications, types of treatment, postoperative rehabilitation and clinical outcomes available in long-term for treatment of this injury.

\section{WhAT IS THE RECOMMENDATION FOR THE CONSERVATIVE TREATMENT OF PCL INJURY?}

The indication of the type of treatment for injuries of the posterior cruciate ligament (PCL) should take into account factors such as the nature of the injury (whether acute or chronic), the type of ligament rupture (avulsion or intrasubstance injury), the degree of laxity (I, II or III), the patient's symptoms, and their occupational or athletic demand (D). ${ }^{8}$ It is well established that PCL repair in bone avulsions leads to an excellent static and functional outcome, but the dilemma persists as to knowing the best option in intrasubstance injuries $(\mathbf{B}){ }^{9}{ }^{9}$

In 1987, Fowler and Messiah performed a prospective study with a minimum follow-up of 2.6 years on 13 patients with intrasubstance PCL injuries submitted to nonoperative treatment. They concluded that grade I and II injuries should be managed non-surgically, while grade III injuries also showed good subjective and functional results after intense physical therapy treatment $(\mathbf{B}){ }^{9}$

Thus, most authors recommend conservative treatment for isolated, low-grade (grades I and II), acute or chronic, mildly symptomatic PCL injuries and for those patients with low demand in daily life or professional activities $\left.(\mathbf{D})^{8,11}(\mathbf{B})\right)^{9,10}$

In 1999, Shelbourne et al. published a prospective study on the natural history of the disease, reporting good functional results from conservative treatment on 133 athletes with isolated PCL injuries after a follow-up of 5.4 years, despite a certain degree of instability (looseness) remaining posterior to the affected knee. Half of the patients returned to the same level of sport activity, while a third returned to the same sport at lower levels $(\mathbf{B}) .{ }^{12}$
Recent studies show that during the radiographic evolution there is some degree of degeneration of the affected knee joint, regardless of the degree of laxity $(\mathbf{D})^{11}$ (B). ${ }^{12}$ This is exactly what Patel et al. demonstrated in 2007 in an average follow-up of 6.9 years on patients with partial or complete grade I and II injuries treated conservatively, with degenerative joint changes reported in $17 \%$ of patients in the medial compartment and $7 \%$ in the patellofemoral compartment $(\mathbf{C}){ }^{13}$

\section{Recommendation: B}

Studies on the natural history of PCL injury show that isolated grade I and II ligament injuries have good functional results with conservative treatment in the long term, even if maintaining a certain degree of laxity, also returning to practice of sport. However, conservative treatment is associated with degenerative joint changes. Nevertheless, there are no randomized prospective studies with conclusive evidence indicating what patients will develop early onset arthrosis and what the magnitude of this disease will be.

\section{When IS SURGical RECONSTRUCTION OF the PCL INDICATED?}

The indications for surgical treatment of PCL injuries also show controversies on the precise indications for ligament reconstruction, due to the lack of randomized clinical trials $(\mathbf{D})^{8}(\mathbf{C}) \cdot{ }^{14}$

Most authors support ligament reconstruction for patients with isolated grade III lesions, tears associated with other knee ligament injuries and bone avulsion injuries $(\mathbf{D})^{8,15,16}(\mathbf{C}) \cdot{ }^{14}$ For patients with high sports performance, isolated ligament reconstruction of the PCL can be conducted on grade II injuries, but this statement depends on the surgeon's experience and the patient's own wishes and factors $(\mathbf{D})^{8}(\mathbf{B}) .^{10}$

Fanelli et al. argue that tibial posteriorization equal to or greater than $8 \mathrm{~mm}$ would be an indication for PCL reconstruction. Factors associated with successful surgical outcomes include the ability to diagnose and properly treat all existing related injuries, the choice of a suitable graft, the positioning of the tibial and femoral tunnels, minimization of the graft curve in its path and an appropriate program of postoperative rehabilitation (D). ${ }^{15}$

\section{Recommendation: B}

Given the absence of randomized prospective studies that demonstrate the precise indication for surgical treatment of PCL injuries, some authors recommend surgical treatment of isolated PCL acute grade II injury for young, ac- 
tive individuals with repairable meniscal injury. However, most authors recommend surgery in grade III injuries when there are other associated injuries and in PCL injuries with bone avulsion.

\section{When SURGery IS INDICATED, IS SINGLE TUNNEL PCL RECONSTRUCTION SIMILAR TO DOUBLE TUNNEL RECONSTRUCTION?}

Transtibial reconstruction surgery using arthroscopy on posterior cruciate ligament (PCL) injuries with the use of a single bundle of ligament graft is called single bundle surgery and has classically been used in symptomatic grade II and grade III PCL injuries $(\mathbf{D}){ }^{16}(\mathbf{B}) \cdot{ }^{17}$ In this technique, the main objective is the reconstruction of the anterolateral portion of the PCL, since it is theoretically primarily responsible for the tibial posterior displacement restriction $(\mathbf{B})^{17}(\mathbf{C}) .^{18,19}$

Surgery involving the use of two bundles of ligament graft is called a double bundle operation and was first proposed as an attempt at better reconstruction of the anatomy of the posterior cruciate ligament, given that with this technique the goal is to reconstruct both the anterolateral portion as well as the posteromedial bundle of the PCL $(\mathbf{D}){ }^{15}$

Despite the theoretical superiority of the double bundle technique over the single bundle technique (reconstitution with greater similarity to PCL anatomy), studies available on the subject show no real difference in longterm results between the techniques. Another major difficulty in comparing the data available on the subject is the wide variety of techniques used by surgeons $(\mathbf{C})^{19}(\mathbf{D})^{20}$ $(\mathbf{A})^{21}(\mathbf{B}) \cdot{ }^{22}$

In an attempt to compare the single and double bundle techniques in isolated PCL reconstruction in 2010, a Korean group retrospectively compared the reconstruction using the same technique (inlay) with single and double bundles, with a minimum follow-up of 60 months. Using the data from clinical scores and radiographic findings, no statistical difference was found between the groups $(\mathbf{B}){ }^{22}$

In 2011, Yoon et al. published a study of 53 patients with isolated PCL injuries, randomly allocated into 2 groups according to the type of PCL reconstruction using allografts. After a minimum follow-up of 2 years, their conclusion was for the objective superiority of double bundle reconstruction, although the subjective and functional analysis showed no difference between groups $(\mathbf{A}){ }^{21}$

\section{Recommendation: A}

Both single and double bundle reconstruction have good functional, objective and subjective results in the long term in patients with isolated PCL injury. The differences found in works with high impact in the literature demonstrate the equality of functional and subjective results, but disagree as to the objective results, tending toward the superiority of the double bundle technique. However, we cannot confirm, based on the available literature, which type of procedure leads to the best results, as there is no agreement between the techniques according to the authors.

\section{Does the MEdial (AM) OR LATERAL (AL) DIRECTION OF THE TIBIAL TUNNEL FOR TRANSTIBIAL PCL RECONSTRUCTION MAKE A DIFFERENCE?}

PCL reconstruction surgery using the transtibial tunnel is traditionally performed by making a tunnel in the anteromedial direction $(\mathbf{A})^{23}(\mathbf{C}){ }^{24}$ During the postoperative follow-up, failure of the graft due to tearing was observed secondary to stress and friction received at the output of the tibial tunnel when performing the curvature toward the femoral condyle $(\mathbf{A})^{23}(\mathbf{C}) .^{24}$ The area of the graft which is found under this tension was named the "Killer turn" in 1992 by Marc Friedman, also known as the curvature of death, based on the consequence of this position for the long-term outcome of surgery $(\mathbf{A})^{23}(\mathbf{D}) .^{25}$

In 2009, Kim et al. published a retrospective study reporting on a follow-up of eight years comparing 60 cases using the anteromedial (23 cases) and anterolateral (37 cases) tunnel techniques, finding significantly different objective results between the tunnels, with superiority of AL. However, when taking into account the clinical results no difference was shown between groups in functional performance (C). ${ }^{24}$

In this same line of reasoning, in 2007, Wong et al. published a prospective study comparing the clinical, functional and radiographic changes between PCL reconstruction with AM and AL tibial tunnel. However, no significant difference was found in outcomes between the directions of the tunnels $(\mathbf{A}){ }^{23}$

Theoretically, the anterolateral tunnel has less chance of developing into tearing of the graft because it does not present an acute angle of curvature at the exit point of the tunnel, thus reducing the "Killer Turn" $(\mathbf{A})^{23}(\mathbf{C})^{24}$ (B) ${ }^{26}$ However, drilling the anterolateral tunnel is a great technical challenge due to the anterolateral surface of the proximal tibia being more vertical to the coronal plane, creating difficulty for the positioning of the drill guide. Another problem consists in the fact that the tunnel is more oblique compared to the anteromedial, causing the orifice of the tibial tunnel to be larger, meaning there may be lower graft fixation strength, resulting in failure secondary to lower loads $(\mathbf{B}) .{ }^{26}$ 


\section{Recommendation: B}

Despite the theoretical (objective) advantages of the anterolateral over the anteromedial technique, clinical studies have not observed differences in functional outcomes in the short and medium term. In addition, several authors mention greater technical difficulty in reconstruction with anterolateral tibial drilling. Thus, there is not enough scientific evidence to choose one technique over the other for single PCL reconstruction.

\section{DOES THE RECONSTRUCTION TECHNIQUE KNOWN AS INLAY HAVE ADVANTAGES OVER THE TRANSTIBIAL TECHNIQUE?}

Several surgical techniques related to the tibial tunnel have emerged in order to improve the outcome of the reconstruction of the PCL. The commonly used transtibial reconstruction restores the posterior stability of the PCL, but makes the graft to bend around the back of the proximal tibia, the so-called killer curve, leading to laxity, abrasion and failure of the graft. The inlay type reconstruction would be an alternative to eliminate this effect, since the graft is placed along with the bone block anatomically in the groove in the posterior region of the tibia, reducing the impact of the tibial bone and consequently decreasing degeneration of the graft. However, short follow-up times do not allow the long term result of this technique to be assessed. For best results, some authors propose the combination of the inlay technique with double bundle reconstruction, arguing that it increases the effectiveness of the reconstruction $(\mathbf{C})^{27,30}(\mathbf{D}) .^{28,29,31,32}$

In 2006 in a retrospective study, Seon and Song compared the two reconstructions in 43 patients, transtibial (21) and inlay (22), showing significant clinical improvement in both groups, but no subjective and radiographic clinical difference $(\mathbf{B}){ }^{33}$

In 2006, MacGillivray et al., in a retrospective study that included a series of 20 cases, evaluated isolated PCL reconstruction with 7 patients undergoing inlay reconstruction and 13 transtibial reconstruction. After a minimum follow-up of two years they concluded that there is no objective and subjective differences between the two techniques $(\mathbf{B}) \cdot{ }^{34}$

In 2009, Kim et al. conducted a prospective study comparing functional scores, clinical and radiographic findings of three PCL reconstruction techniques: transtibial (8), inlay with single bundle (11) and inlay with double bundle (10). They concluded that there was a significant difference in posterior tibial translation between patients undergoing the double bundle inlay technique and the patients submitted to transtibial reconstruction techniques, but without differences between the single bundle inlay and transtibial techniques. No difference was found regarding functional results $(\mathbf{C}){ }^{35}$

\section{Recommendation: B}

Biomechanical studies show no significant differences in ligament laxity and graft strength in the transtibial and inlay techniques, requiring the development of new prospective clinical studies with long-term follow-ups in order to define superiority.

\section{IS USE OF HOMOLOGOUS GRAFTS RECOMMENDED FOR RECONSTRUCTION OF THE PCL?}

In PCL reconstruction, various types of graft are available. These include autologous grafts, homologous grafts and synthetic grafts. Autologous grafts have the advantages of availability, non-rejection and disease transmission, but also have a disadvantage in relation to additional incision, prolonged surgical time and complications such as infection, pain at the donor site and limitation in graft size. Homologous grafts have the advantage of versatility in size and fewer incisions, but entail an additional cost, potential risk of disease transmission and an increased graft failure rate. Currently, little is currently discussed about the use of synthetic grafts, as none has been able to achieve satisfactory clinical results $(\mathbf{D})^{36,37}(\mathbf{B})^{38}(\mathbf{C}){ }^{39}$ Autologous grafts that can be used include the patellar ligament, quadricipital tendon and ischiotibial (flexors) grafts. The homologous graft options include the aforementioned, plus the Achilles tendon and the tendons of the anterior or posterior tibial muscles (D). ${ }^{36}$

Currently, differences have not been described between the results obtained from the repair with autologous grafts and homologous grafts $(\mathbf{B})^{38}(\mathbf{C}) .{ }^{39}$

In a prospective study, Wang et al. compared two groups of patients: 23 isolated PCL reconstructions with homologous grafts and 32 reconstructions with autologous grafts. In a minimum follow-up of 34 months, no significant differences in ligamentous laxity and radiographic changes was found, but there was a higher rate of complications with autologous grafts, including infection, pain at the donor site and complex regional pain syndrome $(\mathbf{B}){ }^{38}$

In 2005, in a case-control study, Ahn et al. evaluated 36 patients divided into two groups, the first being subjected to reconstruction with autologous ischiotibial tendon grafts and the second with Achilles tendon homologous grafts, concluding that there is no statistical difference between the results obtained, evaluated by several tests after a minimum follow-up of 2 years $(\mathbf{C}) .{ }^{39}$ 


\section{Recommendation: B}

The use of homologous grafts is recommended for reconstruction of the PCL. The surgeon is responsible for choosing between the type of graft to be used (homologous or autologous), and the source of this graft according to their experience and individual preference; knowing the limitations and possible complications of each method.

\section{How SHOULd POSTOPERATIVE tReatMent OF PCL RECONSTRUCTION BE CONDUCTED?}

The rehabilitation process in PCL injury is a complementary, yet, essential step for the functional recovery of the knee. Rehabilitation protocols call for the protection of the reconstructed ligament, limiting stress on the graft until it is fully integrated. The safe tensions during rehabilitation exercises are still not known $(\mathbf{C})^{39,40}(\mathbf{D}){ }^{41}$

The key points for rehabilitation include use of extended immobilizer, range of motion gains, load release on the affected limb, muscle strengthening, and proprioceptive training. Furthermore, the use of cryotherapy for reducing swelling and pain can be considered, as well as the use of electrical stimulation to prevent atrophy of the quadriceps and analgesia with local action methods $(\mathbf{D}){ }^{41}$

There is no consensus on the knee flexion gain limit. According to the protocol developed at the Holy House of Mercy in São Paulo (HSCSP), the range of motion respects the angles of $0-70^{\circ}$ in the first four weeks, up to $90^{\circ}$ by the sixth week and, after that, there are progressive gains in amplitude $(\mathbf{C}){ }^{42}$

Quelard et al. advocate gradual passive mobilization, performing a range of $0-60^{\circ}$ in the first six weeks, $0-90^{\circ}$ on the sixth to eighth weeks, and $0-120^{\circ}$ from the eighth week on (D) ${ }^{43}$ McAllister and Hussain $(\mathbf{C})^{44}$ begin mobilization between the third and sixth weeks, Fanelli et al. (D) ${ }^{15,41}$ between the fifth and tenth weeks, and Edson et al. $(\mathbf{D})^{45}$ in the fifth week.

The increase in degree of knee flexion increases tension in the PCL, thus many professionals perform stabilization of the tibia with anterior pressure on the posterior region of the leg to avoid excessive stress on the ligament, as advocated by Irrgang and Fitzgeraldem in their rehabilitation protocol $(\mathbf{D}){ }^{46}$

Early release of loads after isolated PCL reconstruction is common in many protocols, being released as tolerated by the patient as early as in the first week (D) ${ }^{42}$ Quelard et al. (D) $)^{43}$ initially conducted their protocol without weight bearing in the first ten days, progressing to partial weight bearing on the eleventh day until the fifth week and full weight bearing after the sixth week. McAllister and Hussain $(\mathbf{C})^{44}$ do not allow weight bear- ing for three weeks, progressing to partial weight bearing in the fourth and fifth weeks, and full weight bearing in the sixth week. Edson et al. ${ }^{44}$ remained five weeks without weight bearing, progressing to partial weight bearing in the sixth week and full weight-bearing in the tenth week. All authors use braces in extension associated with weight bearing $(\mathbf{C}){ }^{41}$

In relation muscle strengthening, there is a trend in the use of closed kinetic chain exercises (CKC) at the start of the protocols, generating axial compressive forces on the joint, which would decrease the shear forces on the knee, and lead to the simultaneous contraction of the quadriceps and hamstrings, which is desirable in the initial phase of rehabilitation. This is supplemented with open kinetic chain exercises (OKC) in the most advanced phase $(\mathbf{C}){ }^{41}$

According to the national protocol, developed by Cury et al., closed kinetic chain exercises should start from the second week, limiting the range of motion to $0-45^{\circ}$, progressing to open kinetic chain exercises through isometric contraction with an angle of $45-70^{\circ}$ in order to spare the PCL from exaggerated tensions and protect the patellofemoral joint (C). ${ }^{42}$

The protocols usually postpone the introduction of exercises directed at the hamstrings aimed at not tensioning the graft during the initial post-operative period. The group of Brazilian authors either disagrees or has no agreement as to when to start working the hamstrings $(\mathbf{C}) .^{41}$

Proprioceptive training should be undertaken on stable ground with static exercises, progressing to unstable ground with dynamic exercises and increasingly specific to the functional goal. The predicted discharge for sports activities occurs from the sixth postoperative month on $(\mathbf{D})^{15}$ $(\mathbf{C})^{39,40,42,43,44}$ (D) ${ }^{41,45,46}$

\section{Recommendation: C}

There are no level A studies indicating a standard protocol for rehabilitation of PCL injuries, but most authors reach about $70 \%$ success in rehabilitation with different protocols, providing activity levels similar to the pre-injury period.

\section{References}

1. Junior WMW, Bergfeld JA, Parker RD. Evaluation and Treatment of Posterior Cruciate Ligament Injuries. Am J Sports Med. 2004;32(7):1765-75.

2. Allen CR, Kaplan LD, Fluhme D, Harner CD. Posterior Cruciate Ligament Injuries. Current Opinion in Rheumatology. 2002;14:142-49.

3. Fanelli GC, Beck JD, Edson CJ. Current Concepts Review: The Posterior Cruciate Ligament. J Knee Surg. 2010;23(2):61-72.

4. Dowd GSE. Reconstruction of the posterior cruciate ligament: indications and results. J Bone Joint Surg Br. 2004;86-B:480-91. 
5. Matava MJ, Ellis E, Gruber B. Surgical treatment of posterior cruciate ligament tears: evolving technique. J Am Acad Orthop Surg. 2009;17:435-46.

6. Petrigliano FA, McAllister DR. Isolated Posterior Cruciate Ligament Injuries of the Knee. Sports Med Arthrosc Rev. 2006;14(4):206-12.

7. Parolie JM, Bergfel JA. Long-term results of nonoperative treatment of isolated posterior cruciate ligament injuries in the athlete. Am J Sports Med. 1986;14(1):35-38.

8. Vidriero EL, Simon DA, Johnson DH. Initial Evaluation of Posterior Cruciate Ligament Injuries: History, Physical Examination, Imaging Studies, Surgical and Nonsurgical Indications. Sports Med Arthrosc Rev. 2010;18(4):230-37.

9. Fowler PJ, Messieh SS. Isolated Posterior Cruciate Ligament injuries in athletes. Am J Sports Med. 1987;15(6):553-557.

10. Shino K, Horibe S, Nakata K, Maeda A, Hamada M Nakamura N. Conservative Treatment Of Isolated Injuries to the Posterior Cruciate Ligament in Athletes. J Bone Joint Surg. 1995;77(6):895-900.

11. Keller PM, Shelbourne KD, McCarrol JR, Rettig AC. Nonoperatively treated isolated posterior cruciate ligament injuries. Am J Sports Med. 1993;21(1):132-36.

12. Shelbourne KD, Davis TJ, Patel DV. The Natural History of Acute, Isolated, Nonoperatively Treated Posterior Cruciate Ligament Injuries: A Prospective Study. Am J Sports Med. 1999;27(3):276-83.

13. Patel DV, Allen AA, Warren RF, Wickiewicz TL, Simonian PT. The Nonoperative Treatment of Acute, Isolated (Partial or Complete) Posterior Cruciate Ligament-Deficient Knees: An Intermediate-Term Follow-up Study. HSSJ. 2007;3:137-46.

14. Di Felice GS, Lissy M, Haynes P. When to Arthroscopically Repair the Torn Posterior Cruciate Ligament. Clin Orthop Relat Res. 2012;470:861-68.

15. Fanelli GC, Beck JD, Edson CJ. Double Bundle Posterior Cruciate Ligament Reconstruction Surgical Technique and Results. Sports Med Arthrosc Rev. 2010;18(4):242-8

16. Kim YM, Lee CA, Matava MJ. Clinical Results of Arthroscopic Single-bundle Transtibial Posterior Cruciate Ligament Reconstruction. A Systematic Review. Am J Sports Med. 2011;39(2):425-34.

17. Wang CJ, Chen HS, Huang TW. Outcome of Arthroscopic Single Bundle Reconstruction for Complete Posterior Cruciate Ligament Tear. Injury, Int J Care Injured. 2003;34:747-51.

18. Tongel AV, MacDonald PB. Single Bundle Posterior Cruciate Ligament Reconstruction: Surgical Technique and Results. Sports Med Arthrosc Rev. 2010;18(4):238-41.

19. Spiridonov SI, Slinkard NJ, LaPrade RF. Isolated and Combined Grade-III Posterior Cruciate Ligament Tears Treated with Double-bundle Reconstruction with use of Endoscopically Placed Femoral Tunnels and Grafts: Operative Technique and Clinical Results. J Bone Joint Surg Am. 2011;93(19):1773-80

20. Kohen RB, Sekiya JK. Single-Bundle Versus Double-Bundle Posterior Cruciate Ligament Reconstruction. Arthroscopy. 2009;25(12):1470-77.

21. Yoon KH, Bae DK, Song SJ, Cho HJ, Lee JH. A Prospective Randomized Study Comparing Arthroscopy Single-Bundle and Double-Bundle Posterior Cruciate Ligament Reconstruction Preserving Remnant Fibers. Am J Sports Med. 2011;39(3):474-80.

22. Shon OJ, Lee DC, Park CH, Kim WH, Jung KA. A Comparison of Arthroscopically Assisted Single and Double Bundle Tibial Inlay Reconstruction for Isolated Posterior Cruciate Ligament Injury. Clinics in Orthopedic Surgery. 2010;2(2):76-84.

23. Wong T, Wang CJ, Weng LH, Hsu SL, Chou WY, Chen JM et al. Functional outcomes of arthroscopic posterior cruciate ligament reconstruction: comparison of anteromedial and anterolateral trans-tibia approach. Arch Orthop Trauma Surg. 2009;129:315-21.

24. Kim SJ, Chang JH, Kang YH, Song DH, Park KY. Clinical Comparison of Anteromedial Versus Anterolateral Tibial Tunnel Direction for Transtibial Posterior Cruciate Ligament Reconstruction - 2 to 8 Years Follow-Up. Am J Sports Med. 2009;37(4):693-98.
25. Kim SJ, Shin JW, Lee CH, Shin HJ, Kim SH, Jeong JH et al. Biomechanical comparisons of three different tibial tunnel directions in posterior cruciate ligament reconstruction. Arthroscopy. 2005;21(3):286-93.

26. Ahn JH, Bae JH, Lee YS, Choi K, Bae TS, Wang JH. An anatomical and biomechanical comparison of anteromedial and anterolateral approaches for tibial tunnel of posterior cruciate ligament reconstruction. Am J Sports Med. 2009;37(9):1777-83.

27. Papalia R, Osti L, Buono AD, Denaro V, Maffulli N. Tibial inlay for posterior cruciate ligament reconstruction: A systematic review. Knee. 2010;17(4):264-69.

28. Bergfeld JA, McAllister DR, Parker RD, et al. A biomechanical comparison of PCL reconstruction techniques. Am J Sports Med. 2001;29:129-36.

29. Markolf KL, Jemanovic JR, McAllister DR. Cyclic loading of posterior cruciate ligament replacements fixed with tibial tunnel and tibial inlay methods. J Bone Joint Surg Am. 2002;84:518-24.

30. McAllister DR, Markolf KL, Oakes DA, Young CR, McWilliams J. A biomedical comparison of tibial inlay and tibial posterior cruciate ligament reconstruction technique: Graft pretension and knee laxity. Am J Sports Med. 2002;30:312-317.

31. Margheritini F, Mauro CS, Rihn JA, Stabile KJ, Woo SL, Harner CD Biomechanical comparison of tibial inlay versus transtibial techniques for posterior cruciate ligament reconstruction. Am J Sports Med. 2004;32:587-93.

32. Oakes DA, Markolf KL, McWilliams J, Young CR, McAllisterDR. Biomechanical comparison of tibial inlay and tibial tunnel techniques for reconstruction of the posterior cruciate ligament. J Bone Joint Surg Am. 2002;84:938-44.

33. Seon JK, Song EK. Reconstruction of isolated posterior cruciate ligament injuries: a clinical comparison of the transtibial and tibial inlay techniques. Arthroscopy. 2006;22:27-32

34. MacGillivray JD, Stein BE, Park M, et al. Comparison of tibial inlay versus transtibial techniques for isolated posterior cruciate ligament reconstruction: minimum 2-year follow-up. Arthroscopy. 2006;22:320-328.

35. Kim SJ, Kim SH, Kim SG, Kung YP. Comparison of the clinical results of three posterior cruciate ligament reconstruction techniques. J Bone Joint Surg. 2009;91:2543-9.

36. Höher J, Scheffler S, Weiler A. Graft choice and graft fixation in PCL reconstruction. Knee Surg Sports Traumatol Arthrosc. 2003;11:297-306.

37. Frank CB, Jackson DW. The science of reconstruction of the anterior cruciate ligament. J Bone Joint Surg Am. 1997;79:1556-1576.

38. Wang CJ, Chan YS, Weng LH, Yuan LJ, Chen HS. Comparison of autogenous and allogenous posterior cruciate ligament reconstructions of the knee. Injury, Int. J. Care Injured. 2004;35:1279-85.

39. Ahn JH, Yoo JC, Wang JH. Posterior cruciate ligament reconstruction: Doubleloop autograft versus Achilles tendon allograft - Clinical results of a minimum 2-year follow-up. Arthroscopy. 2005;21(8):965-69.

40. Zayni R, Hager J.P, Archbold P, Fournier Y, Quelard B, Chambat P, SonneryCottet B. Activity level recovery after arthroscopic PCL reconstruction: A series of 21 patients with a mean follow-up of 29 months. The Knee. 2011;18:392-5.

41. Fanelli GC. Posterior cruciate ligament rehabilitation: how slow should we go? Arthroscopy. 2008;24(2):234-235.

42. Cury RPL, Kiyomoto HD, Rosal GF, Bryk FF, Oliveira VM, Camargo OPA. Protocolo de reabilitação para as reconstruções isoladas do ligament cruzado posterior. Rev Bras Ortop. 2012;47(4):421-7.

43. Quelard B, Sonnery-Cottet B, Zayni R, Badet R, Fournier Y, Hager JP et al. Isolated posterior cruciate ligament reconstruction: is non-aggressive rehabilitation the right protocol? Orthop Traumatol Surg Res. 2010;96(3):256-62.

44. McAllister DR, Hussain SM. Tibial inlay posterior cruciate ligament reconstruction: surgical technique and results. Sports Med Arthrosc Rev. 2010;18(4):249-53.

45. Edson CJ, Fanelli GC, Beck J. Postoperative rehabilitation of the posterior cruciate ligament. Sports Med Arthrosc Rev. 2010;18(4):275-9.

46. Irrgang JJ, Fitzgerald GK. Rehabilitation of the multiple ligament injured knee. Clinics in Sport Med. 2000;19(3):545-71. 